\title{
Lentiviral-mediated gene silencing of Notch-4 inhibits in vitro proliferation and perineural invasion of $\mathrm{ACC}-\mathrm{M}$ cells
}

\author{
WEI CHEN $^{1 *}$, HUALI ZHANG ${ }^{3 *}$, JIANDONG WANG $^{2}$, GANG CAO $^{1}$, ZHEN DONG $^{1}$, \\ HAN SU $^{1}$, XIAOJUN ZHOU ${ }^{2}$ and SENLIN ZHANG ${ }^{1}$ \\ Departments of ${ }^{1}$ Stomatology, and ${ }^{2}$ Pathology, Nanjing Jinling Hospital, Nanjing University, \\ School of Medicine, Nanjing, Jiangsu; ${ }^{3}$ Department of Orthodontics, School of Stomatology, \\ Fourth Military Medical University, Xi'an, Shaanxi, P.R. China
}

Received November 20, 2012; Accepted January 8, 2013

DOI: $10.3892 /$ or.2013.2317

\begin{abstract}
Salivary adenoid cystic carcinoma (SACC) is a common type of salivary gland cancer. The poor long-term prognosis for patients with SACC is mainly due to local recurrence, perineural invasion (PNI) and distant metastasis. Notch signaling plays a critical role in determining cell fate such as proliferation, differentiation and apoptosis. Accumulating evidence indicates that aberrant Notch-4 expression has a tumor-promoting function in SACC. In the present study, we used lentiviral-mediated RNA interference (RNAi) targeted against Notch-4 to determine the effects of decreased levels of this protein in the human highly metastatic adenoid cystic carcinoma cell line ACC-M. Furthermore, the proliferative capability as well as the PNI potential of the treated cells were observed in vitro. Our studies demonstrated that RNAi directed against Notch-4 markedly decreased Notch-4 gene expression, resulting in the inhibition of cell proliferation, and $G_{0} / G_{1}$ to $S$ phase arrest in ACC-M cells. Knockdown of Notch-4 also resulted in a decrease in the in vitro PNI activity in ACC-M cells. To conclude, RNAi targeting against Notch-4
\end{abstract}

Correspondence to: Professor Senlin Zhang, Department of Stomatology, Nanjing Jinling Hospital, Nanjing University, School of Medicine, 305 East Zhongshan Road, Nanjing, Jiangsu 210002, P.R. China

E-mail: doczhangsl@gmail.com

Professor Xiaojun Zhou, Department of Pathology, Nanjing Jinling Hospital, Nanjing University, School of Medicine, 305 East Zhongshan Road, Nanjing, Jiangsu 210002, P.R. China

E-mail: zh_xjzhou81@yahoo.cn

*Contributed equally

Abbreviations: SACC, salivary adenoid cystic carcinoma; PNI, perineural invasion; siRNA, small interfering RNA; shRNA, short hairpin RNA; TACE, TNF- $\alpha$-converting enzyme; NF- $\kappa B$, nuclear factor- $\kappa \mathrm{B}$; FCM, flow cytometry

Key words: Notch-4, RNAi, proliferation, perineural invasion, adenoid cystic carcinoma induces the suppression of cell growth and inhibition of PNI in vitro in ACC-M cells. Notch-4 may play an important role in regulating proliferation and PNI activity of SACC.

\section{Introduction}

Salivary adenoid cystic carcinoma (SACC) is a common type of salivary gland malignancy, and accounts for $25 \%$ of malignant tumors in the major salivary glands (1) and 50\% in the minor glands (2). The neoplasm is characterized by heterogeneous phenotypic features and persistently progressive biological behavior. The poor long-term prognosis for patients with adenoid cystic carcinoma is mainly due to local recurrence related to perineural invasion (PNI) and delayed onset of distant metastasis, particularly to the lungs $(3,4)$. PNI, a frequent occurrence in SACC, is difficult to be identified clinically and this often prevents complete surgical resection (5). Vrielinck et al (6) reported the relationship between PNI and poor prognosis. PNI has also been observed frequently in other types of cancer such as melanoma, prostate and pancreatic carcinomas as well as head and neck cancers and is recognized as one of the most important prognostic factors (7-11). Due to their predilection for nerves, these cancers are known as 'neurotropic cancers'.

Notch signaling is a pathway highly conserved through evolution which regulates various physiological processes, including stem cell maintenance, differentiation, proliferation and apoptosis $(12,13)$. In mammals, key components of the Notch pathway include four transmembrane receptors (Notch-1, Notch-2, Notch-3 and Notch-4) and five ligands (Dl11, Dl13, Dll4 and Jagged-1, -2) (14,15). Direct binding of a ligand from a signaling cell to a Notch receptor on the membrane of the receiving cell initiates two successive proteolytic cleavages by TACE (TNF- $\alpha$-converting enzyme) and the $\gamma$-secretase/presenilin complex, which ultimately results in the release of the intracellular domain (N-IC). N-IC then translocates into the nucleus and directly interacts with the DNA binding protein $\mathrm{CBF}-1 / \mathrm{Su}(\mathrm{H}) / \mathrm{Lag}-1$ (CSF) that activates the transcription of target genes including the hairy/enhancer-ofsplit (HES-1) (16).

Accumulating evidence strongly indicates that aberrant Notch signaling has a tumor-promoting function in many 
Table I. Oligonucleotide sequences of Notch-4-specific shRNAs.

\begin{tabular}{ll} 
Name & \multicolumn{2}{c}{ Base sequence } \\
\hline shRNA1-F & 5'-CGCGTCCCCGCAGATATGTAAGGACCAGAATTCAAGAGATTCTGGTCCTTACATATCTGCTTTTTGGAAAT-3' \\
shRNA1-R & 5'-CGATTTCCAAAAAGCAGATATGTAAGGACCAGAATCTCTTGAATTCTGGTCCTTACATATCTGCGGGGA-3' \\
shRNA2-F & 5'-CGCGTCCCCCTGCGATAATGCGAGGAAGATTTCAAGAGAATCTTCCTCGCATTATCGCAGTTTTTGGAAAT-3' \\
shRNA2-R & 5'-CGATTTCCAAAAACTGCGATAATGCGAGGAAGATTCTCTTGAAATCTTCCTCGCATTATCGCAGGGGGA-3' \\
shRNA3-F & 5'-CGCGTCCCCAGATATGTAAGGACCAGAATTCAAGAGATTCTGGTCCTTACATATCTTTTTTGGAAAT-3' \\
shRNA3-R & 5'-CGATTTCCAAAAAAGATATGTAAGGACCAGAATCTCTTGAATTCTGGTCCTTACATATCTGGGGA-3' \\
shRNA4-F & 5'-CGCGTCCCCCGATGGACAGAACTGCTCATTCAAGAGATGAGCAGTTCTGTCCATCGTTTTGGAAAT-3' \\
shRNA4-R & 5'-CGATTTCCAAAAACGATGGACAGAACTGCTCATCTCTTGAATGAGCAGTTCTGTCCATCGGGGGA-3' \\
NC-F & 5'-CGCGTCCCCTTCTCCGAACGTGTCACGTTTCAAGAGAACGTGACACGTTCGGAGAATTTTTGGAAAT-3' \\
NC-R & 5'-CGATTTCCAAAAATTCTCCGAACGTGTCACGTTCTCTTGAACGTGACACGTTCGGAGAAGGGGA-3'
\end{tabular}

shRNA1-4 indicates the oligonucleotide sequence of Notch-4. NC, negative control. Underlined section indicates the forward and reverse target sequence; bold section is the restriction enzyme cutting site, including 5'-ACGCGT-3' (MIul) and 5'-ATCGAT-3' (CIal). shRNA1 and 3 also include the restriction enzyme cutting site of EcoRI (5'-GAATTC-3', italicized section).

types of tumors, and Notch signaling may be a promising target for cancer treatment. A role for Notch signaling in salivary gland adenocarcinoma cells has been suggested which proposes that 5'-nitro-indirubinoxime (5'-NIO) induces $\mathrm{G}_{0} / \mathrm{G}_{1}$ cell cycle arrest and apoptosis by the downregulation of Notch-1 signaling (17). Notch-1 cross-talk has also been reported in other major cell growth and apoptotic regulatory pathways through modulating the activity of the transcription factor, for example, nuclear factor (NF)- $\mathrm{B}$ and $\mathrm{Wnt} / \beta$-catenin signaling $(18,19)$. Notch signaling may contribute to squamous cell carcinogenesis, and it is considered as a candidate marker for squamous cell carcinomas of the head and neck (HNSCC) (20). It was reported that the Notch signaling pathway also contributes to the drug resistance of cancer cells. Inhibition of Notch signaling was found to prevent drug resistance and enhanced chemosensitivity in human myeloma, breast cancer and HNSCC (21-23). In SACC, a recent study suggested that Notch-4 activation contributes to SACC metastasis (24).

In our previous microarray study, Notch- 4 was found to play a potential important role in the pathobiology of SACC associated with PNI (25). Thus, we tested our hypothesis on whether knockdown of Notch-4 by short hairpin RNA (shRNA) inhibits the in vitro proliferation and PNI in ACC-M cells.

To examine our hypothesis, we silenced Notch-4 expression in a human highly metastatic SACC cell line, ACC-M (26), by lentiviral vector-mediated RNA interference (RNAi) technology, and evaluated the effect of Notch-4 on cell growth, cell cycle distribution and cell PNI activity in ACC-M cells. Our data showed that Notch-4 RNAi had antiproliferative activity by modulating $G_{0} / G_{1}$ and $S$ cell cycle regulators. The knockdown of Notch-4 expression by lentiviral vectormediated RNAi reduced the PNI activity in vitro in SACC cells. These results suggest that Notch-4 plays an important role in regulating the in vitro growth, proliferation and PNI of ACC-M cells. The suppression of Notch- 4 may be a potential therapeutic strategy for SACC.

\section{Materials and methods}

Cell lines and cell culture condition. ACC-M and 293T cells were kindly provided by the Department of Oral Biology, School of Stomatology, Fourth Military Medical University, China. The two types of cells were cultured in Dulbecco's modified Eagle's medium (DMEM) (Invitrogen Corp. Carlsbad, CA, USA) supplemented with $10 \%$ of fetal bovine serum (FBS) (Gibco, Invitrogen), $2.05 \mathrm{mM}$ of L-glutamine, $100 \mathrm{~g} / \mathrm{ml}$ of penicillin and $100 \mu \mathrm{g} / \mathrm{ml}$ of streptomycin at $37^{\circ} \mathrm{C}$ in $5 \% \mathrm{CO}_{2}$.

Preparation of Notch-4 lentiviral vectors. Lentiviral vector system (pLenOR-THM, pMDLg/pRRE, pRSV-Rev, pMD2.G) was purchased from Innovation Biotechnology Co., Ltd. (Shanghai, China). Referencing siRNA design strategy $(27,28)$, we selected sites of the gene (NM_004557.3) cDNA sequence and determined the specific sequence by BLAST. Four pairs of siRNA and one negative control were designed and synthesized. As shown in Table I, each pair contained a unique 21-nt (shRNA1 and 2) or 19-nt (shRNA3 and 4) double-stranded human Notch-4 sequence that is presented as an inverted complementary repeat and separated by a loop of a 9-nt spacer. DNA oligonucleotides (Table I) targeting Notch-4 were synthesized and inserted into the MIul and CIal site of the linearized lentiviral-shRNA expression vector according to the manufacturer's instructions. They were incorporated into a pLenOR-THM expression plasmid. The successful ligation was confirmed by the restrictive cleavage and sequenced for an additional verification (Fig. 1). The recombinant vectors were named pLenOR-Notch-4-shRNA1, 2, 3 and 4.

The recombinant vector was then mixed with virus packaging mix, which including pMDLg/pRRE (HIV-1 gag/pol component), pRSV-Rev (a binding site for the Rev protein which facilitates export of the RNA from the nucleus) and pMD2.G (VSV-G component), packed and transfected by Lipofectamine $^{\mathrm{TM}} 2000$ (Invitrogen) into 293T cells. Viral supernatant was harvested $48 \mathrm{~h}$ after transfection, filtered 


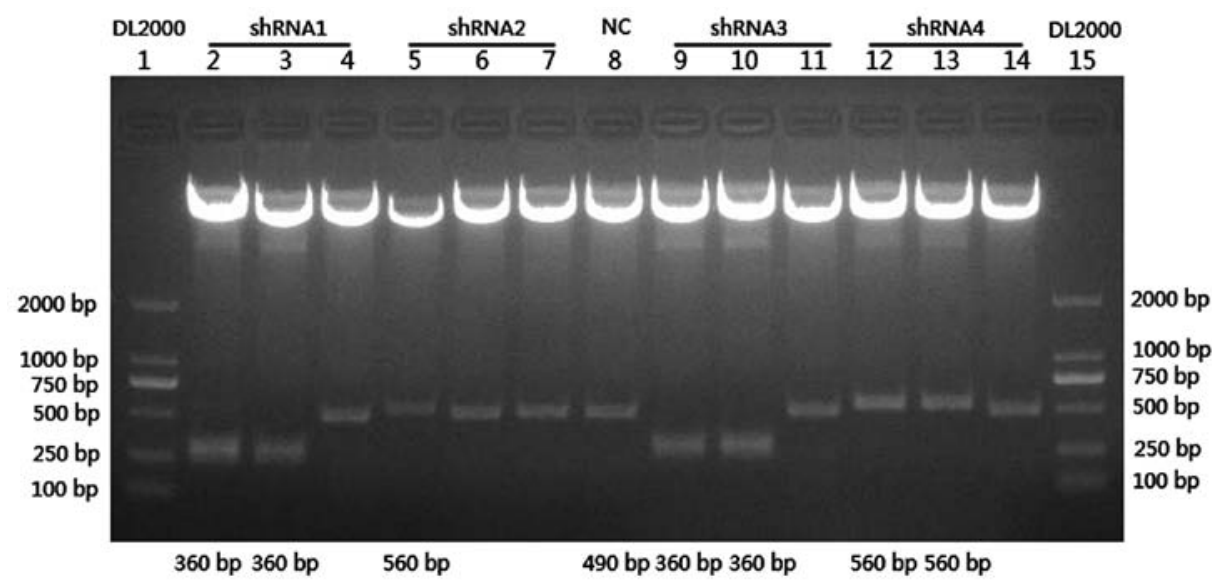

Figure 1. Validation results of the recombinant vector by EcoRI and XbaI double-digestion. Lanes 1 and 15 were Marker DL2000. Lanes 2, 3 and 4 were three clones of shRNA1, which contains the restriction enzyme cutting site of EcoRI (5'-GAATTC-3'). Lanes 2 and 3 were confirmed as positive clones (360 bp). Lanes 5, 6 and 7 were three clones of shRNA2, and lane 5 was confirmed as a positive clone (560 bp). Lanes 9, 10 and 11 were three clones of shRNA3, which also contains the restriction enzyme cutting site of EcoRI. Lanes 9 and 10 were confirmed as positive clones ( $360 \mathrm{bp}$ ). Lanes 12, 13 and 14 were three clones of shRNA4, and lanes 12 and 13 were confirmed as positive clones (560 bp). Lane 8 was a negative control (490 bp).

through a $0.45-\mu \mathrm{m}$ cellulose acetate filter and frozen at $-70^{\circ} \mathrm{C}$. Virus titer was detected by a 96-well plate dilution method and flow cytometry.

Lentiviral transfection and construction of stable silenced cell lines. Approximately 2x10 ACC-M cells/well were plated in 6-well plates. Twenty-four hours later, ACC-M cells were transfected with $1 \times 10^{7}$ specific or negative control lentiviral vectors (multiplicity of infection of 25) containing $500 \mu \mathrm{l}$ enhancing transfection solution (Innovation Biotechnology Co., Ltd.) and $8 \mu \mathrm{g} / \mathrm{ml}$ Polybrene ${ }^{\circledR}$ (Sigma, St. Louis, MO, USA). At $24 \mathrm{~h}$ post-transfection, the medium was replaced by normal medium containing $10 \%$ FBS and antibiotics. After $96 \mathrm{~h}$ post-transfection, the transfected cells were observed under a fluorescence microscope (Leica). As the lentiviral vector contains a GFP expression cassette, the cell transfection rate was directly observed, which reached $90 \%$.

Quantitative RT-PCR. Quantitative RT-PCR (qRT-PCR) for Notch-4 transcripts (shRNA1, 2, 3 and 4) in the ACC-M cell lines was performed. ACC-M cells transfected by zero-loaded lentiviral vector and untreated ACC-M cells were taken as the positive and the negative control separately. Total RNA was extracted from $1 \times 10^{6}$ cells with TRIzol reagent (Invitrogen). Samples of total RNA $(1 \mu \mathrm{g})$ were reverse-transcribed into cDNA using a kit according to the manufacturer's instructions (Qiagen, Valencia, CA, USA). qRT-PCR was performed with QuantiTect SYBR Green PCR Master Mix (Qiagen) using the Rotor-Gene RG-3000 Real-Time Thermal Cycler (Corbett Research, Sydney, Australia) and Rotor-Gene software version 6.0 .

Each reaction mixture contained $10 \mu \mathrm{l}$ SYBR-Green Master Mix, $0.5 \mu 1$ of each sense and antisense primer, $0.5 \mu 1$ cDNA template supplemented with water to a final volume of $20 \mu 1$. The specific primers of Notch-4 (forward, 5'-TCAACACT CCTGGCTCCTTCAACT-3', and reverse, 5'-AGAGGCAC TCATTGTGATCAGCCT-3') were amplified as follows: $94^{\circ} \mathrm{C}$ for $3 \mathrm{~min}$ and 40 cycles at $94^{\circ} \mathrm{C}$ for $30 \mathrm{sec}$, followed by $61.1^{\circ} \mathrm{C}$ for $30 \mathrm{sec}, 72^{\circ} \mathrm{C}$ for $20 \mathrm{sec}$, then ended with $72^{\circ} \mathrm{C}$ for $10 \mathrm{~min}$ for elongation. Human 18S gene was amplified as the internal control (forward, 5'-CGGCTACCACATCCAAGGAA-3' and reverse, 5'-GCTGGAATTACCGCGGCT-3'). Target genes and the $18 \mathrm{~S}$ gene were amplified in the same reaction. Each sample was performed in triplicate. Comparative quantification was determined using the $2^{-\Delta \Delta \mathrm{Ct}}$ method.

Western blot analysis. Cells were washed twice with cold phosphate-buffered saline and lysed on ice in buffer containing protease inhibitors. Equal amounts of protein $(20 \mu \mathrm{g} / \mathrm{lane})$ from the cell lysates were electrophoresed on $10 \%$ acrylamide gels. After SDS-PAGE, proteins were transferred to a polyvinylidene difluoride membrane. The membrane was incubated for $2 \mathrm{~h}$ in PBS plus $0.1 \%$ Tween 20 and 5\% non-fat skim milk to block non-specific binding. Subsequently, the membrane was incubated for $2 \mathrm{~h}$ with an antibody against Notch-4 (R\&D Systems, Minneapolis, MN, USA). After washing, proteins were visualized using an ECL detection kit with the appropriate HRP-conjugated secondary antibody (Amersham Pharmacia Biotech, Piscataway, NJ, USA). The membranes were stripped and probed with monoclonal antibodies for GAPDH for loading control as per standard protocols. The experiment was repeated three times to confirm the results.

Proliferation assay. The MTT (3-[4,5-dimethylthiazol-2-yl] -2,5-diphenyltetrazolium bromide) colorimetric assay was performed to assess the cell proliferation of the transfected cells. Briefly, the cells were plated in 96-well plates at a density of $10^{3}$ cells/well. Then for 8 days, every $24 \mathrm{~h}$, a batch of cells were stained with $20 \mu \mathrm{l}$ sterile MTT dye $(5 \mathrm{mg} / \mathrm{ml}$; SigmaAldrich) at $37^{\circ} \mathrm{C}$ for $4 \mathrm{~h}$. The culture medium was then removed, and $150 \mu \mathrm{l}$ of dimethyl sulphoxide (DMSO) was added and thoroughly mixed in for $10 \mathrm{~min}$. Spectrometric absorbance at $490 \mathrm{~nm}$ was measured using a microplate reader. Each group consisted of three wells.

Flow cytometric analysis. Different cell cycle phases $\left(\mathrm{G}_{0} / \mathrm{G}_{1}, \mathrm{~S}\right.$ or $\mathrm{G}_{2} / \mathrm{M}$ phase) are characterized by different DNA contents. Fluorescence dye propidium iodide (PI) binds with DNA 
strongly at a ratio of $1: 1$, and hence the DNA contents of cell cycle phases are reflected by varying PI fluorescent intensities. Stable transfected ACC-M cells, $1 \times 10^{6}$, were harvested by trypsinization and fixed in $70 \%$ ice-cold $\left(4^{\circ} \mathrm{C}\right)$ ethanol for $2 \mathrm{~h}$. Cell pellets were resuspended in $1 \mathrm{mg} / \mathrm{ml}$ RNase solution (Sigma-Aldrich) for $30 \mathrm{~min}$ at $37^{\circ} \mathrm{C}$ and subsequently in $0.1 \mathrm{mg} / \mathrm{ml}$ PI solution (Sigma-Aldrich) at $4^{\circ} \mathrm{C}$ for $1 \mathrm{~h}$ in the dark. Cell cycle analysis was performed on a flow cytometer (Beckman Coulter, Inc., Fullerton, CA, USA).

In vitro perineural invasion assay. The inhibitory effect of RNAi on the PNI ability of ACC-M cells in vitro was demonstrated in modified Boyden chambers. Transwell invasion chambers containing polycarbonate filters $(8-\mu \mathrm{m})$ (Millipore Corp., Billerica, MA, USA) were coated on the upper surface with Matrigel basement membrane (BD Biosciences, San

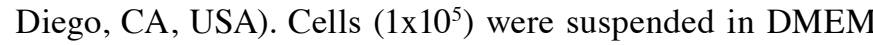
supplemented with $1 \%$ fetal bovine serum and added to the upper chamber. The lower chamber contained $600 \mu \mathrm{l}$ supernatant of 24 h-cultured RSC96 cells (a rat Schwann cell line, purchased from the Cell Bank for Type Culture Collection, Chinese Academy of Sciences) as a chemoattractant to simulate the perineural surrounding environment (29,30). Cells were incubated for $12 \mathrm{~h}$ at $37^{\circ}$ in $5 \% \mathrm{CO}_{2}$ incubator. At the end of the incubation, the cells on the upper surface of the filter were completely removed by wiping with a cotton swab. The filters were then fixed in methanol and stained with hematoxylin and eosin. Cells that had invaded the Matrigel and had reached the lower surface of the filter were counted under a light microscope at a magnification of $x 400$. We chose five fields of vision and counted the numbers of the invaded cells on the lower surface of the filter. The assay was performed in triplicate.

Statistical analysis. Results are expressed as means \pm standard deviation (SD). Statistical analysis was performed using SPSS 17.0 statistical software (SPSS Inc., Chicago, IL, USA). Data were tested for statistical significance using analysis of variance (ANOVA). Normally distributed, continuous variables were compared using one-way ANOVA. When ANOVA produced a significant difference between groups, multiple comparisons of group means were performed using the Bonferroni procedure with a type I error adjustment. All P-values were two-sided, and significance was defined as $\mathrm{P}<0.05$.

\section{Results}

Lentiviral vector-mediated RNAi of Notch-4 causes effective and specific downregulation of Notch-4 expression. The knockdown efficiencies of different Notch-4-specific shRNAs in ACC-M cells were first evaluated using qRT-PCR. Relative Notch-4 mRNA levels in individual stable transfectants were normalized against mRNA levels of an internal control gene, human 18S, performed in the same run. As shown in Fig. 2A, cells transfected with pLenOR-Notch-4-shRNA2, 3 and 4 showed a significantly reduced transcription of Notch- 4 mRNA when compared with that of the negative control ACC-M cells and the positive control vector ACC-M Mock, respectively $(\mathrm{P}<0.01)$, but there was no significant mRNA transcription
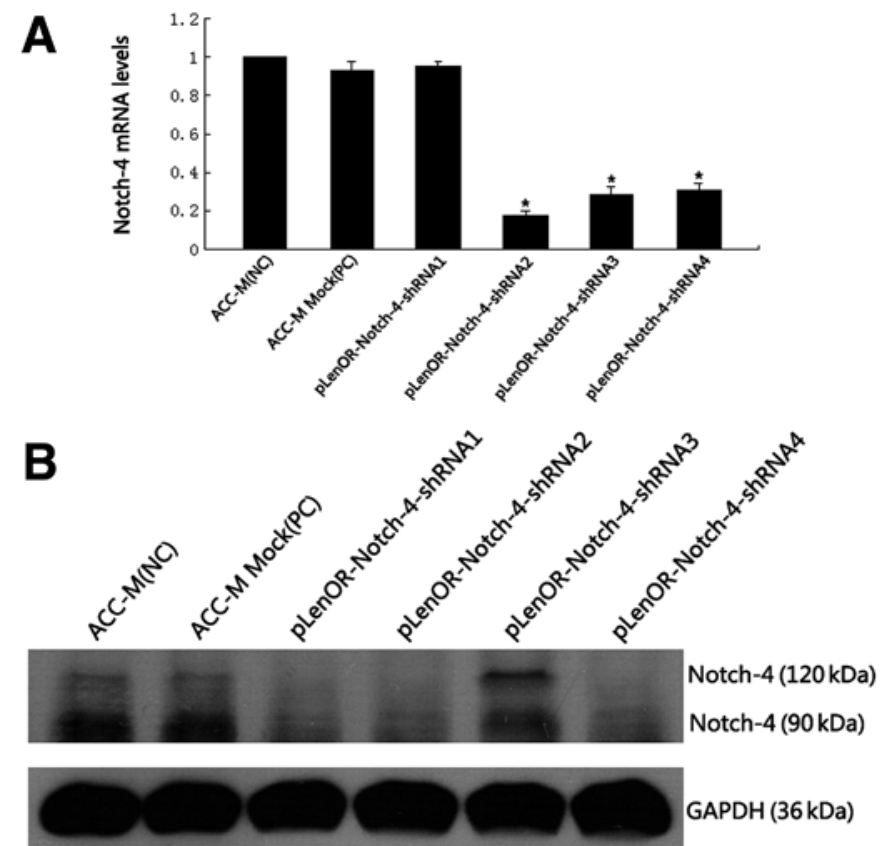

Figure 2. Confirmation of Notch-4 expression in different clones by qRT-PCR and western blot analysis in ACC-M cells. (A) Notch-4 mRNA levels were determined by qRT-PCR. Relative fold induction for the Notch-4 mRNA (means $\pm \mathrm{SD}$ ) in mock and Notch-4 siRNA-transfected cells is presented relative to the expression in parental ACC-M cells. Cells transfected with pLenOR-Notch-4-shRNA2, 3, 4 showed a significantly reduced transcription of Notch-4 mRNA when compared with that of the negative control ACC-M cells and the positive control vector ACC-M Mock, respectively, while there was no significant reduction in mRNA transcription in cells transfected with pLenOR-Notch-4-shRNA1 ("P<0.01 compared with ACC-M). (B) Western blot analysis for Notch-4 protein expression in the indicated cell lines. GAPDH was used as a loading control. The Notch-4 protein is a $\sim 210 \mathrm{kDa}$ heterodimer. The Notch-4 protein was split by the lysate, so therefore the western blot analysis detected the intracellular and extracellular domain of Notch-4 protein in the cells. Western blot analysis revealed a decreased expression of Notch- 4 protein in the ACC-M cells transfected with pLenORNotch-4-shRNA1, 2, 4, while cells transduced by pLenOR-Notch-4-shRNA3 showed no notable reduced expression of Notch-4 protein compared with that of the negative control ACC-M cells and the positive control vector ACC-M Mock. These results revealed that the most effective vector was pLenOR-Notch-4-shRNA2. ACC-M, high metastatic potential control used as a negative control; ACC-M Mock, mock transfection control used as the positive control) pLenOR-Notch-4-shRNA1, 2, 3 and 4 represent the four different clones, respectively.

reduction in cells transfected with pLenOR-Notch-4-shRNA1. The cells transfected with pLenOR-Notch-4-shRNA2 showed the most significant inhibition of Notch-4 mRNA levels.

The Notch-4 protein is a heterodimer $210 \mathrm{kDa}$, and the Notch-4 protein was split by the lysate, thus our western blot analysis detected the intracellular and extracellular domain of Notch-4 protein in the cells. In addition, western blot analysis (Fig. 2B) showed reduced expression of Notch-4 protein in the ACC-M cells transfected with pLenOR-Notch-4-shRNA1, 2, 4, while cells transduced by pLenOR-Notch-4-shRNA3 showed no notable decreased expression of Notch-4 protein compared with that of the negative control ACC-M cells and the positive control vector ACC-M Mock. The above results demonstrated that the expression of Notch-4 was specifically and effectively downregulated by Notch-4 RNAi, allowing its application for the subsequent experiment. For the sake of convenience, we chose the most effective vector (pLenOR-Notch-4-shRNA2)- 


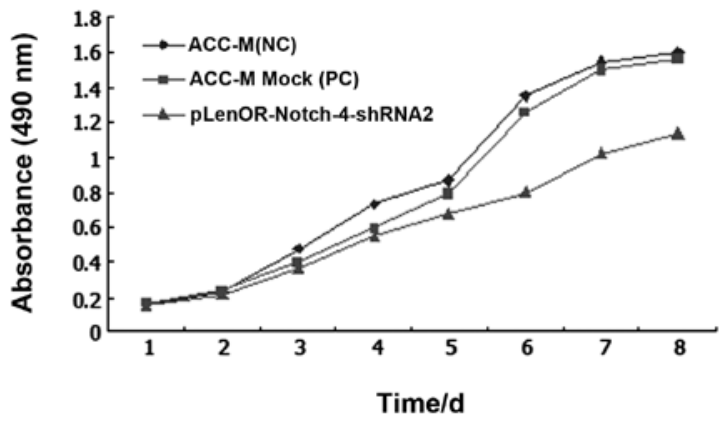

Figure 3. Gene silencing of Notch-4 reduces cell proliferation in ACC-M cells. Cell proliferation was analyzed using the MTT assay as described in Materials and methods. Cells were monitored for 8 days, and the average OD490 ( \pm SD) for each cell line is shown. Each group contained three wells. Statistical analysis was performed with repeated measurement ANOVA. Significant changes in the proliferation curves were observed between the Notch-4 RNAi (pLenOR-Notch-4-shRNA2) cells and negative control (ACC-M) or positive control (ACC-M Mock) cells $\left(\mathrm{P}_{1,3}<0.01, \mathrm{P}_{2,3}<0.01\right.$ ). Each data point represents the mean of three independent wells. 1, ACC-M; 2, ACC-M Mock; 3, pLenOR-Notch-4-shRNA2.

transfected cells, positive and negative control groups to study the cell biological behavior.

Gene silencing of Notch-4 reduces cell proliferation in vitro. The proliferative activity of tumor cells is important in the invasion/metastasis of tumors. To examine whether the knock- down of Notch-4 expression has any effect on cell growth, an MTT cell proliferation assay was performed. Under the same cell culture conditions, the proliferative activity of the pLenOR-Notch-4-shRNA2-transfected cells, negative and positive control cells was almost similar for the first $24 \mathrm{~h}$. With time-lapse, the cells with Notch- 4 gene silencing grew more slowly when compared with the control cells. Among the three groups, Notch-4 RNAi (pLenOR-Notch-4-shRNA2) cells showed decreased cell proliferation, when compared to the negative control (ACC-M) and mock-transfected (ACC-M Mock) cells, supporting the role of Notch-4 in the cell growth of ACC-M cells $(\mathrm{P}<0.01)$ (Fig. 3).

We next used flow cytometry (FCM) to study the effect of Notch-4-specific shRNA on the cell cycle distribution in ACC-M cells. The negative control group (ACC-M) and positive control group (ACC-M Mock) resulted in a cell cycle distribution of $\sim 53$ and $46 \%$ of the cells in the $S$ and $\mathrm{G}_{2} / \mathrm{M}$ phases. In the pLenOR-Notch-4-shRNA2 group, the proliferation index value (PrI) of cycling cells (combined total number of cells in the $\mathrm{S}$ and $\mathrm{G}_{2} / \mathrm{M}$ phases) was decreased to $\sim 39 \%$, with a concomitant increase in the number of cells in the $\mathrm{G}_{0} / \mathrm{G}_{1}$ phase (Table II) (Fig. 4). Significant decreases in PrI were found in the pLenOR-Notch-4-shRNA2-transfected cells compared with negative and positive control cells $(\mathrm{P}<0.01)$. There was no significant difference in PrI between that of the negative and positive control transfectant cells $(\mathrm{P}>0.05)$. These findings indicate that knockdown of Notch- 4 expression
A
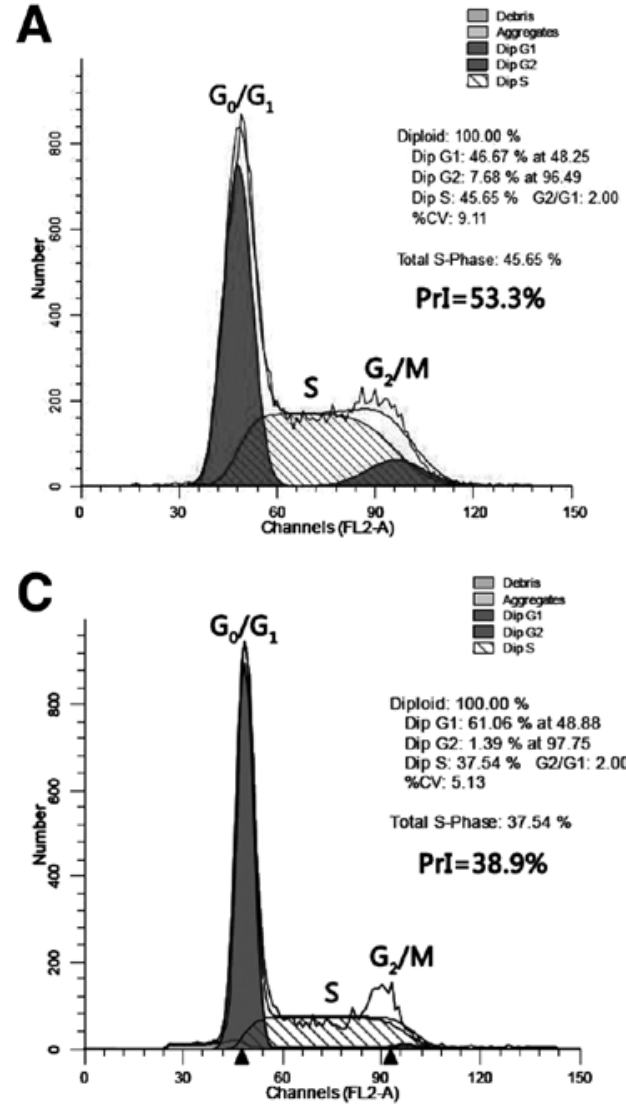

B

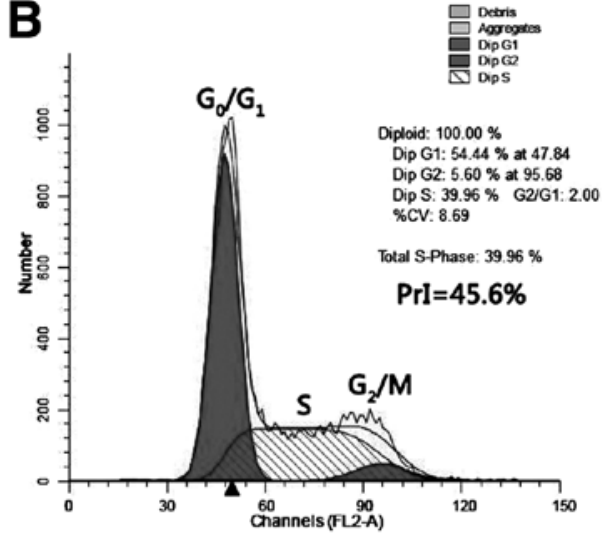

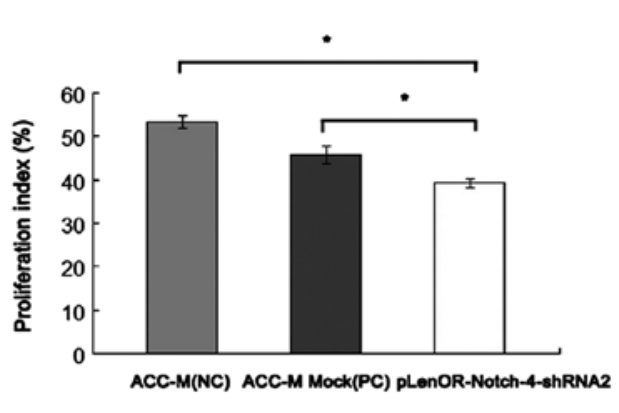

Figure 4. Representative examples of FCM analysis of the ACC-M cells after Notch-4 specific inhibition. (A) ACC-M (NC), (B) ACC-M Mock (PC), (C) pLenOR-Notch-4-shRNA2. Statistical analysis was performed with one-way ANOVA. Significant decreases in PrI were found in the pLenOR-Notch-4shRNA2 stable transfected cells compared with the negative or positive control cells ( $\left.{ }^{*} \mathrm{P}_{\mathrm{A}, \mathrm{C}}<0.01,{ }^{*} \mathrm{P}_{\mathrm{B}, \mathrm{C}}<0.01\right)$. No significant difference in PrI was noted between the ACC-M and ACC-M Mock cells $\left(\mathrm{P}_{\mathrm{A}, \mathrm{B}}>0.05\right)$. PrI, proliferation index value. 
Table II. FCM analysis of the cell cycle of ACC-M cells after Notch-4-specific inhibition.

\begin{tabular}{lcccc}
\hline Cell group & $\mathrm{G}_{0} / \mathrm{G}_{1}(\%)$ & $\mathrm{S}(\%)$ & $\mathrm{G}_{2} / \mathrm{M}(\%)$ & Proliferation index $(\%)$ \\
\hline ACC-M (NC) & $46.7 \pm 1.45$ & $45.7 \pm 0.96$ & $7.6 \pm 0.50$ & $53.3 \pm 1.45$ \\
ACC-M Mock (PC) & $54.2 \pm 2.06$ & $40.2 \pm 1.27$ & $5.5 \pm 0.80$ & $45.8 \pm 2.06$ \\
pLenOR-Notch-4-shRNA2 & $60.8 \pm 1.12$ & $37.8 \pm 1.16$ & $1.5 \pm 0.10$ & $39.2 \pm 1.12^{\mathrm{a}}$ \\
\hline
\end{tabular}

${ }^{\mathrm{a}} \mathrm{P}_{1,3}<0.01, \mathrm{P}_{2,3}<0.01, \mathrm{P}_{1,2}>0.05 .1$, ACC-M (NC); 2, ACC-M Mock (PC); 3, pLenOR-Notch-4-shRNA2.

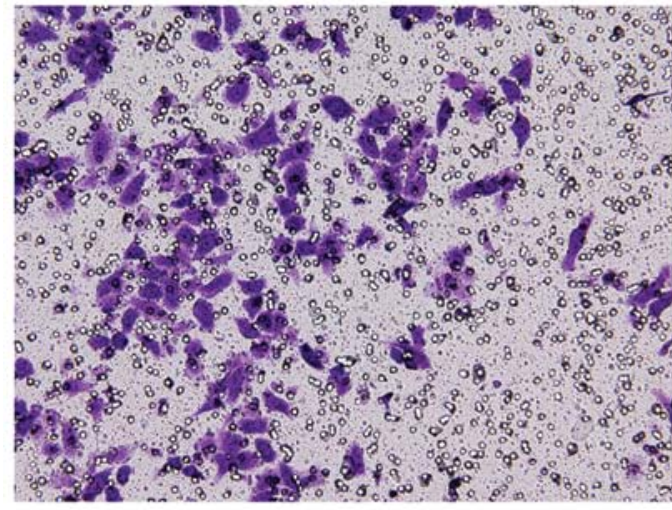

ACC-M(NC)

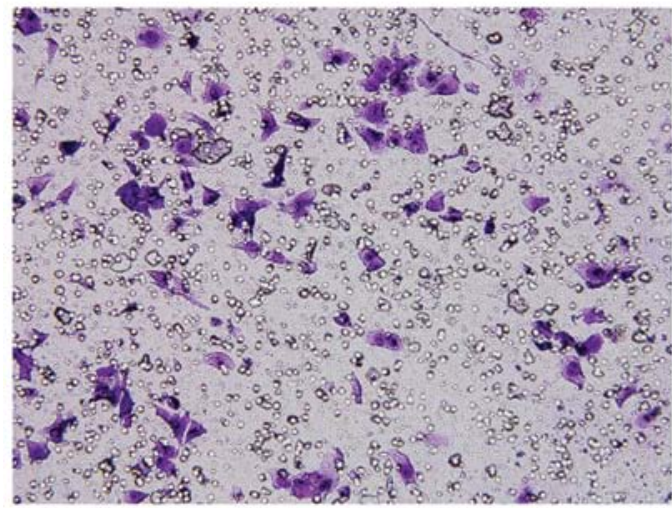

pLenOR-Notch-4-shRNA2

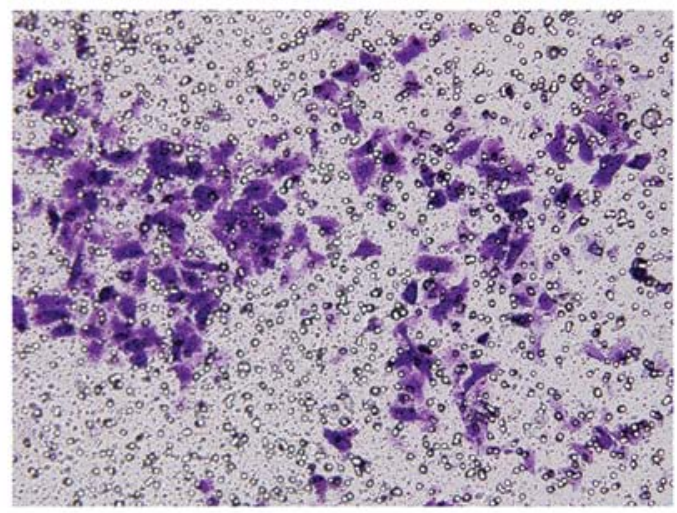

ACC-M Mock(PC)

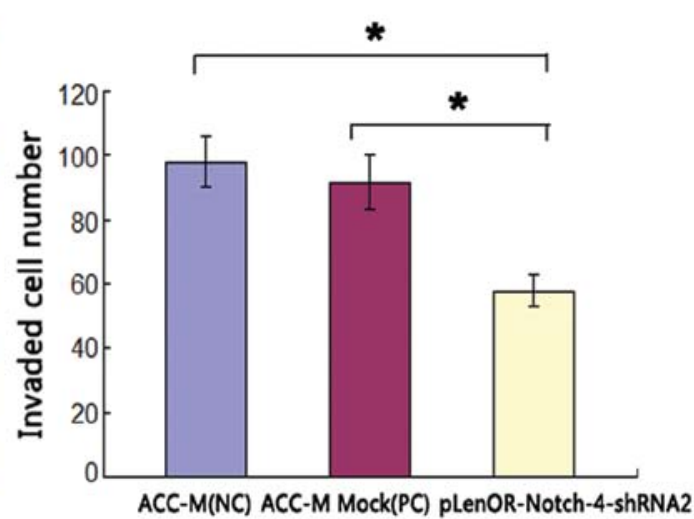

Figure 5. Effects of Notch-4-specific shRNA on Matrigel perineural invasion of ACC-M cells. Matrigel PNI was evaluated using modified Boyden chambers as described in Materials and methods. The numbers of cells migrated were evaluated in three fields for each experimental group and averaged. The average invaded cell number of the groups ACC-M (NC), ACC-M Mock (PC) and pLenOR-Notch-4-shRNA2 were 98.0 $\pm 7.98,91.5 \pm 8.46$ and 57.8 \pm 4.95 , respectively. Statistical analysis was performed with one-way ANOVA. $\mathrm{P}_{1,3}<0.01, \mathrm{P}_{2,3}<0.01, \mathrm{P}_{1,2}>0.05$. 1, ACC-M (NC); 2 , ACC-M Mock (PC); 3, pLenOR-Notch-4shRNA2 (magnification, $\mathrm{x} 400)$.

may inhibit proliferation of ACC-M cells by modulating $\mathrm{G}_{0} / \mathrm{G}_{1}$ and $\mathrm{S}$ cell cycle regulators.

Gene silencing of Notch-4 inhibits in vitro perineural invasion ability of ACC-M cells. PNI activity of the effective Notch4-specific shRNA transfectant (pLenOR-Notch-4-shRNA2) was assayed in vitro by modified Boyden chambers. Cells transfected with pLenOR-Notch-4-shRNA2 showed much lower PNI activities compared with the negative and the positive controls $(\mathrm{P}<0.01)$. There was no significant difference in in vitro $\mathrm{PNI}$ ability between the negative and positive control transfectant cells $(\mathrm{P}>0.05)$ (Fig. 5). These data confirm that the knockdown of Notch-4 expression inhibits in vitro PNI activity of ACC-M cells.

\section{Discussion}

The gene encoding the Notch receptor was discovered almost 90 years ago, and gained its name because partial loss of Notch function resulted in notches in the wing margins of Drosophila (31). It only became apparent some years later that the Notch signaling pathway has been conserved throughout evolution. The Notch signaling pathway plays a pivotal role in several cell functions, such as cell fate decisions, cell proliferation, differentiation and cell death during development and postnatal life in species as diverse as Drosophila, worms and vertebrates (32-36). In the mammalian system, there are four Notch receptors (Notch-1, Notch-2, Notch-3 and Notch-4) and five known ligands (D111, Dll3, Dll4 and 
Jagged-1, -2) (14,15). Notch is a cellular fate determinant and can induce cell proliferation and/or differentiation, depending on the cellular environment (37). A recent study showed that targeting Notch-1 and Notch-4 may provide a new therapeutic strategy for triple-negative and possibly other breast cancer subtypes (38). In SACC, Notch-4 may play a key role in SACC metastasis, and inhibition of Notch-4 gene expression may have potential therapeutic application in treating metastatic patients (24).

Perineural invasion (PNI), is a typical biological behavior of SACC, which may prevent a complete surgical resection (5). PNI is associated with poor prognosis in SACC patients (6). In our previous study, we established the gene expression profile of SACC associated with PNI by combining the use of laser capture microdissection (LCM) and cDNA microarray. In the profile, Notch-4 was notably overexpressed in the PNI cell group, and this was verified by qRT-PCR (25). Thus, we hypothesized that inhibition of Notch-4 gene expression may reduce in vitro proliferation and PNI in ACC-M cells.

To understand the biological function of Notch-4 in SACC, we examined the effects of a decreased expression of Notch-4 in a human highly metastatic SACC cell line, ACC-M, using a lentiviral-mediated RNAi system. RNAi uses the phenomenon by which double-strand RNA induces potent and specific inhibition of eukaryotic gene expression through the degradation of complementary messenger RNA (mRNA) and is functionally similar to the processes of post-transcriptional gene silencing (39). In the past few years, siRNA and shRNA have been widely used to silence the expression of many target genes, and both methods have had great achievement, but the silencing effect lasts for less than 2 weeks. New systems based on lentiviral vectors have provided new solutions to achieving stable shRNA-mediated knockdown $(40,41)$.

In the present study, we used a lentiviral-mediated RNAi method to obtain effective knockdown of the Notch-4 gene in ACC-M cells and constructed stable silencing clones.

Here, we designed four shRNAs targeted at the Notch-4 gene and successfully transfected them into ACC-M cells Among the four designed shRNAs, the cells transfected with pLenOR-Notch-4-shRNA2 showed the most significant inhibitory effect as determined by qRT-PCR and western blot analysis. The results indicated that lentiviral-mediated RNAi of Notch-4 silenced the expression of Notch-4 effectively and specifically in ACC-M cells.

We next examined the consequence of ACC-M cells transfected with Notch-4-specific shRNA. The proliferation of the ACC-M cells in which the Notch-4 gene was knocked down was inhibited compared with that of the positive or negative control. In the FCM study, the Notch-4-knockdown ACC-M cells showed an arrest in $\mathrm{G}_{0} / \mathrm{G}_{1}$-to-S transition, suggesting growth inhibition of these cells (Table II). Therefore, Notch-4 may be a positive regulator of cell growth to promote a mitogenic signal, which then enhances cell proliferation of ACC-M cells.

In the present study, the silencing of Notch-4 in ACC-M cells inhibited the cell PNI activity in vitro. This result was consistent with the results of the MTT assay and FCM analysis which revealed that silencing of Notch- 4 by lentiviral-mediated RNAi inhibited the cell proliferation in vitro by modulating $\mathrm{G}_{0} / \mathrm{G}_{1}$ and $\mathrm{S}$ cell cycle regulators. The finding may also be associated with the metastasic ability of Notch-4 in SACC and breast cancer $(24,38)$.

SACC is a common subtype of salivary gland malignancy, and it has an important biological behavior for PNI. It is urgent to develop new therapeutic strategies for SACC. In this report, the knockdown of Notch-4 expression by lentiviral-mediated RNAi successfully inhibited the malignant behaviors of ACC-M cells, particularly PNI ability in vitro, implicating that Notch-4 may be a new candidate target gene for the treatment of SACC.

\section{Acknowledgements}

This study was supported by grants from the National Natural Science Foundation of China (Project no. 81102051), the Natural Science Foundation of Jiangsu Province (Project no. BK2011659) and the Youth Foundation of Nanjing Jinling Hospital (Project no. 2010Q009).

\section{References}

1. Renehan A, Gleave EN, Hancock BD, Smith P and McGurk M: Long-term follow-up of over 1000 patients with salivary gland tumours treated in a single centre. Br J Surg 83: 1750-1754, 1996.

2. Anderson JN Jr, Beenken SW, Crowe R, Soong SJ, Peters G, Maddox WA and Urist MM: Prognostic factors in minor salivary gland cancer. Head Neck 17: 480-486, 1995.

3. Van der Wal JE, Becking AG, Snow GB and van der Waal I: Distant metastases of adenoid cystic carcinoma of the salivary glands and the value of diagnostic examinations during followup. Head Neck 24: 779-783, 2002.

4. Ramer N, Wu H, Sabo E, Ramer Y, Emanuel P, Orta L and Burstein DE: Prognostic value of quantitative p63 immunostaining in adenoid cystic carcinoma of salivary gland assessed by computerized image analysis. Cancer 116: 77-83, 2010.

5. Van der Wal JE, Snow GB and van der Waal I: Intraoral adenoid cystic carcinoma. The presence of perineural spread in relation to site, size, local extension, and metastatic spread in 22 cases. Cancer 66: 2031-2033, 1990.

6. Vrielinck L, Ostyn F, van Damme B, van den Boqaert W and Fossion E: The significance of perineural spread in adenoid cystic carcinoma of the major and minor salivary glands. Int $\mathbf{J}$ Oral Maxillofac Surg 17: 190-193, 1988.

7. Newlin HE, Morris CG, Amdur RJ and Mendenhall WM: Neurotropic melanoma of the head and neck with clinical perineural invasion. Am J Clin Oncol 28: 399-402, 2005.

8. Ayala GE, Dai H, Ittmann M, Li R, Powell M, Frolov A, Wheeler TM, Thompson TC and Rowley D: Growth and survival mechanisms associated with perineural invasion in prostate cancer. Cancer Res 64: 6082-6090, 2004.

9. Pour PM, Bell RH and Batra SK: Neural invasion in the staging of pancreatic cancer. Pancreas 26: 322-325, 2003.

10. Faqan JJ, Collins B, Barnes L, D'Amico F, Myers EN and Johnson JT: Perineural invasion in squamous cell carcinoma of the head and neck. Arch Otolaryngol Head Neck Surg 124: 637-640, 1998.

11. Rahima B, Shingaki S, Nagata M and Saito C: Prognostic significance of perineural invasion in oral and oropharyngeal carcinoma. Oral Surg Oral Med Oral Pathol Radiol Endod 97: 423-431, 2004.

12. Go MJ, Eastman DS and Artavanis-Tsakonas S: Cell proliferation control by Notch signaling in Drosophila development. Development 125: 2031-2040, 1998.

13. Shelly LL, Fuchs C and Miele L: Notch-1 inhibits apoptosis in murine erythroleukemia cells and is necessary for differentiation induced by hybrid polar compounds. J Cell Biochem 73: 164-175, 1999.

14. Bigas A, Martin DI and Milner LA: Notch1 and Notch2 inhibit myeloid differentiation in response to different cytokines. Mol Cell Biol 18: 2324-2333, 1998.

15. Mumm JS and Kopan R: Notch signaling: from the outside in. Dev Biol 228: 151-165, 2000. 
16. Stockhausen MT, Sjölund J and Axelson H: Regulation of the Notch target gene Hes-1 by TGFalpha induced Ras/MAPK signaling in human neuroblastoma cells. Exp Cell Res 310: 218-228, 2005

17. Yoon JH, Kim SA, Kwon SM, Park JH, Park HS, Kim YC, Yoon JH and Ahn SG: 5'-Nitro-indirubinoxime induces G1 cell cycle arrest and apoptosis in salivary gland adenocarcinoma cells through the inhibition of Notch-1 signaling. Biochim Biophys Acta 1800: 352-358, 2010.

18. Yao J, Duan L, Fan M, Yuan J and Wu X: Notch1 induces cell cycle arrest and apoptosis in human cervical cancer cells: involvement of nuclear factor kappa B inhibition. Int J Gynecol Cancer 17: 502-510, 2007.

19. Duan L, Yao J, Wu X and Fan M: Growth suppression induced by Notch1 activation involves Wnt- $\beta$-catenin down-regulation in human tongue carcinoma cells. Biol Cell 98: 479-490, 2006.

20. Leethanakul C, Patel V, Gillespie J, Pallente M, Ensley JF, Koontongkaew S, Liotta LA, Emmert-Buck M and Gutkind JS: Distinct pattern of expression of differentiation and growthrelated genes in squamous cell carcinomas of the head and neck revealed by the use of laser capture microdissection and cDNA arrays. Oncogene 19: 3220-3224, 2000.

21. Nefedova Y, Sullivan DM, Bolick SC, Dalton WS and Gabrilovich DI: Inhibition of Notch signaling induces apoptosis of myeloma cells and enhances sensitivity to chemotherapy. Blood 111: 2220-2229, 2008

22. Zang S, Chen F, Dai J, Guo D, Tse W, Qu X, Ma D and Ji C: RNAi-mediated knockdown of Notch-1 leads to cell growth inhibition and enhanced chemosensitivity in human breast cancer. Oncol Rep 23: 893-899, 2010.

23. Gu F, Ma Y, Zhang Z, Zhao J, Kobayashi H, Zhang L and Fu L: Expression of Stat 3 and Notch1 is associated with cisplatin resistance in head and neck squamous cell carcinoma. Oncol Rep 23: 671-676, 2010

24. Ding LC, She L, Zheng DL, Huang QL, Wang JF, Zheng FF and Lu YG: Notch-4 contributes to the metastasis of salivary adenoid cystic carcinoma. Oncol Rep 24: 363-368, 2010.

25. Chen W, Zhang HL, Shao XJ, Jiang YG, Zhao XG, Gao X, Li JH, Yang J, Zhang YF, Liu BL and Sun MY: Gene expression profile of salivary adenoid cystic carcinoma associated with perineural invasion. Tohoku J Exp Med 212: 319-334, 2007.

26. Guan XF, Qiu WL, He RG and Zhou XJ: Selection of adenoid cystic carcinoma cell clone highly metastatic to the lung: an experimental study. Int J Oral Maxillofac Surg 26: 116-119, 1997.

27. Tuschl T: Expanding small RNA interference. Nat Biotechnol 20: 446-448, 2002.

28. Reynolds A, Leake D, Boese Q, Scaringe S, Marshall WS and Khvorova A: Rational siRNA design for RNA interference. Nat Biotechnol 22: 326-330, 2004.
29. Wang L, Sun M, Jiang Y, Yang L, Lei D, Lu C, Zhao Y, Zhang P, Yang Y and Li J: Nerve growth factor and tyrosine kinase A in human salivary adenoid cystic carcinoma: expression patterns and effects on in vitro invasive behavior. J Oral Maxillofac Surg 64: 636-641, 2006

30. Chen W, Zhang HL, Jiang YG, Li JH, Liu BL and Sun MY: Inhibition of CD146 gene expression via RNA interference reduces in vitro perineural invasion on ACC-M cell. J Oral Pathol Med 38: 198-205, 2009.

31. Mohr OL: Character changes caused by mutation of an entire region of a chromosome in Drosophila. Genetics 4: 275-282, 1919.

32. Miele L and Osborne B: Arbiter of differentiation and death: Notch signaling meets apoptosis. J Cell Physiol 181: 393-409, 1999.

33. Cheng P and Gabrilovich D: Notch signaling in differentiation and function of dendritic cells. Immunol Res 41: 1-14, 2008.

34. Baker NE: Notch signaling in the nervous system. Pieces still missing from the puzzle. Bioessays 22: 264-273, 2000.

35. Artavanis-Tsakonas S, Rand MD and Lake RJ: Notch signaling: cell fate control and signal integration in development. Science 284: 770-776, 1999.

36. Milner LA and Bigas A: Notch as a mediator of cell fate determination in hematopoiesis: evidence and speculation. Blood 93: 2431-2448, 1999.

37. Kopan R and Ilagan MX: The canonical Notch signaling pathway: unfolding the activation mechanism. Cell 137: 216-233, 2009.

38. Clementz AG, Rogowski A, Pandya K, Miele L and Osipo C: Notch-1 and Notch-4 are novel gene targets of PEA3 in breast cancer: novel therapeutic implications. Breast Cancer Res 13: R63, 2011.

39. Zamore PD, Tuschl T, Sharp PA and Bartel DP: RNAi: doublestranded RNA directs the ATP-dependent cleavage of mRNA at 21 to 23 nucleotide intervals. Cell 101: 25-33, 2000.

40. Yamamoto T, Miyoshi H, Yamamoto N, Yamamoto N, Inoue J and Tsunetsugu-Yokota Y: Lentivirus vectors expressing short hairpin RNAs against the U3-overlapping region of HIV nef inhibit HIV replication and infectivity in primary macrophages. Blood 108: 3305-3312, 2006.

41. Desclaux M, Teigell M, Amar L, Vogel R, Gimenez Y Ribotta M, Privat A and Mallet J: A novel and efficient gene transfer strategy reduces glial reactivity and improves neuronal survival and axonal growth in vitro. PLoS One 4: e6227, 2009. 